\title{
Global Motion Processing by Populations of Direction- Selective Retinal Ganglion Cells
}

\author{
Jon Cafaro, ${ }^{1}$ Joel Zylberberg, ${ }^{2}$ and ${ }^{\circledR}$ Greg D. Field ${ }^{1}$ \\ ${ }^{1}$ Department of Neurobiology, Duke University, Durham, North Carolina, 27710, and ${ }^{2}$ Department of Physics and Astronomy, York University, \\ Toronto, Ontario, M3J 1P3
}

\begin{abstract}
Simple stimuli have been critical to understanding neural population codes in sensory systems. Yet it remains necessary to determine the extent to which this understanding generalizes to more complex conditions. To examine this problem, we measured how populations of direction-selective ganglion cells (DSGCs) from the retinas of male and female mice respond to a global motion stimulus with its direction and speed changing dynamically. We then examined the encoding and decoding of motion direction in both individual and populations of DSGCs. Individual cells integrated global motion over $\sim 200 \mathrm{~ms}$, and responses were tuned to direction. However, responses were sparse and broadly tuned, which severely limited decoding performance from small DSGC populations. In contrast, larger populations compensated for response sparsity, enabling decoding with high temporal precision $(<100 \mathrm{~ms})$. At these timescales, correlated spiking was minimal and had little impact on decoding performance, unlike results obtained using simpler local motion stimuli decoded over longer timescales. We use these data to define different DSGC population decoding regimes that use or mitigate correlated spiking to achieve high-spatial versus high-temporal resolution.
\end{abstract}

Key words: decoding; motion; multielectrode array; population code; retina; vision

\section{Significance Statement}

ON-OFF direction-selective ganglion cells (ooDSGCs) in the mammalian retina are typically thought to signal local motion to the brain. However, several recent studies suggest they may signal global motion. Here we analyze the fidelity of encoding and decoding global motion in a natural scene across large populations of ooDSGCs. We show that large populations of DSGCs are capable of signaling rapid changes in global motion

\section{Introduction}

Sensory systems encode and decode information across populations of neurons. Understanding such population codes is fundamental to understanding the function of neural circuits and sensory processing (Pouget et al., 2000; Panzeri et al., 2015). Population codes are likely optimized for natural sensory stimuli but they are often probed using simple and artificial stimuli (Felsen et al., 2005; Fitzgerald and Clark, 2015). Such simplifications may limit an understanding of population codes and neural

\footnotetext{
Received Mar. 9, 2020; revised June 9, 2020; accepted June 12, 2020.

Author contributions: J.C., J.Z., and G.D.F. designed research; J.C. and G.D.F. performed research; J.C., J.Z., and G.D.F. analyzed data; J.C. and G.D.F. wrote the paper.

This work was supported by the Sloan Research Fellowship in Neuroscience (J.Z.), the Canadian Institute for Advanced Research (J.Z.), the Azrieli Global Scholar Award for Learning in Machines and Brains (J.Z.), the Canada Research Chairs program (J.Z.), the Whitehead Scholars Program at Duke University (G.D.F.), and the National Eye Institute/National Institutes of Health Grant R01 EY024567 (to G.D.F.). We thank G. Awatramani, N.-Y. Jun, B. Murphy-Baum, L. Osborne, S. Roy, and X. Yao for comments on drafts of this manuscript. We also thank T. Mrsic-Flogel and F. lacaruso for providing a movie from a camera attached to the head of a mouse.

The authors declare no competing financial interests.

Correspondence should be addressed to Greg D. Field at field@neuro.duke.edu

https://doi.org/10.1523/JNEUROSCI.0564-20.2020

Copyright $\odot 2020$ the authors
}

function in ethological contexts. In this article, we examine a canonical population code, direction coding in mammalian $\mathrm{ON}$ OFF direction-selective ganglion cells (ooDSGCs), in the context of global motion of a natural scene.

In the mammalian retina, there are four types of ooDSGCs, each tiling space with their dendritic and receptive fields (Barlow et al., 1964; Devries and Baylor, 1997; Demb, 2007; Vaney et al., 2012; Morrie and Feller, 2016). These types differ primarily in their preferred direction of motion, which are organized along four cardinal axes (Oyster and Barlow, 1967; Vaney, 1994; Kay et al., 2011; Trenholm et al., 2013; Yao et al., 2018). Direction is encoded across the four types by their relative firing rates. This produces a population code for direction that is relatively invariant to object speed and contrast (Nowak et al., 2011; Zylberberg et al., 2016). ooDSGCs have been largely considered responsible for signaling local motion, because global motion attenuates (but does not eliminate) their responses (Vaney et al., 2001; Chiao and Masland, 2003; Olveczky et al., 2003; Hoggarth et al., 2015). A separate class of DSGCs, so-called ON DSGCs (oDSGCs), are minimally attenuated by global motion, and have thus been assumed to play a dominant role in signaling global motion (Oyster, 1968; Simpson et al., 1988). Correspondingly, previous 
studies examining the fidelity and accuracy of the ooDSGC population code have focused on local motion and artificial stimuli that are decoded at relatively long timescales (Fiscella et al., 2015; Zylberberg et al., 2016). These studies largely pointed toward a high-fidelity code that utilizes correlated activity in nearby ooDSGCs to signal the direction of local motion. However, recent work indicates that ooDSGCs may be organized to encode self-motion, a global motion signal (Kay et al., 2011; Dhande et al., 2013; Sabbah et al., 2017). This motivates an examination of ooDSGC individual and population responses under conditions in which the stimulus is a natural scene moving globally and dynamically on the retina. It also motivates understanding how the direction of global motion can be decoded from populations of mammalian DSGCs and the extent to which concepts applicable to decoding local motion at long timescales apply to decoding global motion at shorter, and perhaps more behaviorally relevant, timescales.

To study DSGC responses, we recorded simultaneously the spiking activity from hundreds of retinal ganglion cells (RGCs) using a large-scale multielectrode array (MEA). We distinguished DSGCs from other RGCs based on their responses to drifting gratings (Elstrott et al., 2008; Yao et al., 2018). We then projected dynamically moving natural images onto the retina: the motion is "dynamic" because the direction and speed are not constant. Individual ooDSGCs and oDSGCs exhibited similar encoding of dynamic global motion stimuli: they both integrated and low-pass filtered direction signals over a timescale of $\sim 200 \mathrm{~ms}$; they were both broadly tuned; and they both exhibited similar spike rates. Importantly, both ooDSGC and oDSGCs exhibited little trial-to-trial variability in their responses to dynamic global motion, indicating that while the responses were sparse, they were reliable.

We then used our more complete populations of ooDSGCs to examine the limitations inherent in decoding dynamic global motion signals from small and large ooDSGC populations. For a local quartet of ooDSGCs (each with a different preferred direction), determining the direction of global motion was marginally better than chance at short timescales $(\sim 100 \mathrm{~ms})$. Decoding accuracy was improved by longer temporal integration of ooDSGC signals, however this is only an effective decoding strategy when changes in motion direction are infrequent and when the animal does not need to rapidly respond to a change in the motion signal. When motion direction changes frequently, large populations of ooDSGCs are needed to accurately and rapidly $(<100 \mathrm{~ms})$ decode the direction of global motion. Large populations of ooDSGCs are available for decoding at no cost to spatial resolution because the nature of the motion signal is global. Furthermore, the short integration times used when decoding large populations result in largely uncorrelated population activity, which is counter to previous results decoding local motion at long timescales (Franke et al., 2016; Zylberberg et al., 2016). This limits the impact of correlated spiking on decoding accuracy in a dynamic global motion context. Thus, large populations of nearly-independent ooDSGC signals integrated over short timescales enables rapid decoding of global motion.

\section{Materials and Methods}

\section{Experimental design and statistical analysis}

Retinas were removed and recorded from C57BL/6J and CBA/CaJ $\mathrm{x}$ $\mathrm{C} 57 \mathrm{BL} / 6 \mathrm{~J}$ mice between the ages of one month and one year. The strains and sexes showed no differences to the results reported in this study; thus, data were pooled. 21 Animals were used and data from 10 animals (four male and six female) were used in this manuscript. Data were selected based on measurement stability $(<10 \%$ change in spontaneous activity and sensitivity) over the duration of the experiment. All statistical tests and associated information (e.g., $p$ values) are noted where appropriate in the text. Mice were used in accordance with the Duke University Institutional Animal Care and Use Committee.

\section{Retina dissection}

Retina dissection was optimized to maintain response sensitivity. Mice were dark-adapted overnight, euthanized via decapitation, eyes were enucleated, and a piece of retina $\left(\sim 1-2 \mathrm{~mm}^{2}\right)$ was isolated from the pigmented epithelium (Yao et al., 2018). Retina isolation was performed in Ames solution (room temperature) bubbled with $95 \% \mathrm{O}_{2}$ and $5 \% \mathrm{CO}_{2}$. All procedures were performed in the dark under IR light. The retina was isolated from the dorsal half of the eye (identified from vasculature) to increase the fraction of $\mathrm{M}$-opsin expressed in the cones for better overlap with the spectrum of the visual display.

\section{MEA recording, spike sorting, cell position determination}

Electrical activity was measured from RGCs on a MEA (Fig. 1). Spikes were identified, sorted into individual cell clusters, and soma positions on the MEA were estimated as previously described (Litke et al., 2004; Yao et al., 2018). Electrical activity was measured from RGCs using a hexagonal large-scale MEA, which was $\sim 490 \mu \mathrm{m}$ along an edge with 30 $\mu \mathrm{m}$ spacing between 519 electrodes (Field et al., 2010). Retinas were held against the MEA with a permeable membrane and were perfused with Ames solution $\left(34^{\circ} \mathrm{C}\right)$ bubbled with $95 \% \mathrm{O}_{2}$ and $5 \% \mathrm{CO}_{2}$.

Electrical activity was analyzed offline to identify and sort spikes into individual cell clusters (Shlens et al., 2006). Briefly, on each electrode, spikes were identified by a voltage threshold and voltage waveforms were concatenated across the six surrounding electrodes. These concatenated waveforms were parameterized with principal components analysis (PCA) and clustered with a mixture of gaussians model, providing putative cell assignments. Putative cells were analyzed if their spike time autocorrelation showed $<10 \%$ refractory period violations and $<25 \%$ spike time correlation with a cell identified on a nearby electrode, indicating spikes were from a single and uniquely identified neuron.

Soma position on the array was used to identify quartets (Fig. 2) and pairwise distances between cells (Fig. 3A). Soma position was estimated from the electrical image (EI) on the array (Li et al., 2015; Yao et al., 2018). The EI consisted of the average voltage on each electrode preceding a spike (Petrusca et al., 2005; Field et al., 2009). The position of the soma was taken as the center of mass of the EI.

\section{Visual stimulus}

The retina was stimulated at photopic light levels $\left(8000 \mathrm{Rh}^{\star} / \mathrm{s}\right)$ with a $\gamma$-corrected OLED display (SVGA + XL Rev3 from eMagin). Three types of visual stimuli were presented to the retina and controlled via custom software written in MATLAB using the MGL library (https://gru. stanford.edu). First, drifting gratings, at two different temporal frequencies, were used to identify ooDSGCs (see below, RGC classification). Second, natural images, taken from the van Hateren image database (van Hateren and van der Schaaf, 1998), were presented to probe RGC responses to global motion in natural images. The original images in this database spanned $\sim 25.6 \times 17.1^{\circ}$ of visual angle and were $1535 \times 1024$ pixels. During presentation in these experiments, an aperture of $400 \times 400$ pixels was used to display the image to the retina. The optics of the microscope generated a scale factor between pixel size and microns of $4 \mu \mathrm{m} /$ pixel. Thus, the apertured images covered an area of $1.6 \times 1.6 \mathrm{~mm}$, or $\sim 48 \times 48^{\circ}$ of visual angle on the retina (given $\sim 30^{\circ}$ of visual angle per $1 \mathrm{~mm}$ in mouse retina). This corresponded to a magnification factor of $\sim 4 \times$ between the original images and their presentation on the retina. Natural images were presented in two different stimulus protocols; using either dynamic or static velocity. Finally, natural movies from a camera mounted to the head of a mouse (from the lab of Thomas Mrsic-Flogel) and a cat (Betsch et al., 2004) were used to further test response sparsity (Fig. 1Eii, movies 1 and 2, respectively).

In the dynamic velocity protocol, the same image was presented in the same orientation on every frame. The average frame rate was $40 \mathrm{~Hz}$ $(\sim 25 \mathrm{~ms} /$ frame). As noted above, a $400 \times 400$ pixel region (aperture) 



Figure 1. DSGCs integrate the direction of global motion over time and respond sparsely with broad tuning to natural images. A, Natural image presented (top) and displaced according to $\Delta \mathrm{X}$ and $\Delta \mathrm{Y}$ (bottom). Yellow scale bar: 400 monitor pixels, $1.6 \mathrm{~mm}$ on retina. $\boldsymbol{B}$, STA of $\Delta \mathrm{X}$ and $\Delta \mathrm{Y}$ for single example 00DSGC and an oDSGC (inset). C, Direction (Ci) and magnitude (Cii) of motion calculated from STA for all 00DSGCS in a single retinal recording. Color coded according to preferred direction from grating stimulus. Inset to Cii, Distribution of image displacement velocities filtered by the 00DSGC STAs on a log scale (gray bars, left axis) and the static nonlinearities associated with each ooDSGC input-output relationship on a linear scale (solid curves, right axis). D, Spike times during $5 \mathrm{~s}$ of dynamic global motion stimulus for all $00 D S G(s$ in a single retinal recording; color indicates preferred direction (see inset) determined from drifting gratings. Inset shows direction preferences in visual coordinates (S: superior; P: posterior). E, Probability distributions for DSGCS in a single retinal recording using a jittered image (EI) and for ooDSGC and ODSGCS in two different retinal recordings using two different jittered images and two different natural movies (Eii). Movies included video from a camera mounted on a mouse (movie 1) and a cat (movie 2). $\boldsymbol{F}$, Spike raster of a single 00DSGC (Fi) or oDSGC (Fii) simultaneously recorded over several repeated presentations of the same jittered image. $\mathbf{G}$, Gray bars show the distribution of the difference between preferred direction from STA (panel $C_{i}$ ) and the direction of motion $\sim 100 \mathrm{~ms}$ (peak of STA) preceding each spike. Histogram includes data from all ooDSGC cells and all spikes from a single retina ( $n=49 \mathrm{RGCS}$ ). Red bars show a similar distribution, but the direction preceding the spike is calculated by convolving the stimulus by the temporal weighting function of the STA (panel Cii). Similar results were observed in a second retinal recording. The inset shows the same analysis for all oDSGCs in the same recording.

was used to display a portion of the image to the retina. The initial position of the image was centered with respect to the aperture. This position was considered $(0,0)$ in an $(\mathrm{X}, \mathrm{Y})$ coordinate system. Subsequent image positions shifted the portion of the image that was displayed through the mask. These positions over time, $\mathrm{X}(\mathrm{t})$ and $\mathrm{Y}(\mathrm{t})$ were produced by sampling from two Gaussian distributions (both with mean $=0$ and $\mathrm{SD}=100$ pixels). These samples were then smoothed by time averaging over a sliding window of 100 frames. This operation caused the X-Y positions of the image to be correlated in time. However, the changes in position over time, $\Delta \mathrm{X}(\mathrm{t})$ and $\Delta \mathrm{Y}(\mathrm{t})$, were uncorrelated at temporal frequencies above $0.5 \mathrm{~Hz}$ and were normally distributed. The SD of the distribution of these displacements $[\Delta \mathrm{X}(\mathrm{t})$ and $\Delta \mathrm{Y}(\mathrm{t})]$ was $\sim 20 \mu \mathrm{m}$, corresponding to $\sim 800 \mu \mathrm{m} / \mathrm{s}$ along a single axis. If the edge of the original image ever reached the edge of the aperture, the edge of the image was prevented on the next frame from entering the aperture, restricting the image to either remain stationary along that direction or to move away from the aperture on subsequent frames. In practice, these events occurred rarely in our experiments and did not perturb the statistically independent image displacements over time. A single dynamically moving image was presented for $60 \mathrm{~min}$.

In the static direction protocol, the image was drifted in a single direction at $\sim 1080 \mu \mathrm{m} / \mathrm{s}$ for $\sim 4 \mathrm{~s}$ before a new direction or image was presented. The image was reoriented for each direction with its longer edge parallel to the direction of motion. Six different images were presented at eight different directions, spread equally across $360^{\circ}$.

\section{RGC classification}

oDSGCs and ooDSGCs were identified based on their responses to square wave gratings $(960 \mu \mathrm{m} /$ cycle $)$ drifted in eight different directions and two different speeds $(1.0$ and $0.25 \mathrm{~Hz}$ ). Responses to each grating were quantified by total spike number generated during the $8 \mathrm{~s}$ each grating was presented. Cells were first clustered as DSGCs, then separated as $\mathrm{ON}-\mathrm{OFF}$ and $\mathrm{ON}$ cells, and then grouped by their preferred direction (Yao et al., 2018). Direction-selective cells were clustered by their direction-selective indices (DSIs; Ravi et al., 2018) at each grating speed using a 2-dimensional Gaussian mixture model. This method avoided setting an arbitrary threshold on DSI. Cells were then clustered by hand using the ratio of their response vector magnitudes for fast and slow gratings. Cells that maintained or increased their vector magnitudes for faster gratings were identified as ON-OFF. This process is based on the speed tuning curve differences between ON and ON-OFF DSGCs in mouse retina (Yao et al., 2018). Finally, ooDSGCs were clustered by hand based on the direction of their vector sum. Clustering by their preferred direction was only used to color code Figure $1 C$ and did not contribute to decoding (see below, Optimal linear and optimal quadratic estimators).

\section{Optimal linear and optimal quadratic estimators}

An optimal linear estimator (OLE) and optimal quadratic estimator (OQE) were used to estimate the direction of stimulus motion from a set of RGCs responses (Figs. 2, 3, 4) (Salinas and Abbott, 1994; Shamir and Sompolinsky, 2004). To do this, the OLE and OQE weight and sum the responses for each RGC:

$$
\hat{\mathbf{d}}=\overrightarrow{\mathbf{r}}(t+d t) * \mathbf{W},
$$

$\hat{\mathbf{d}}$ is a 2-dimensional vector consisting of $\mathrm{X}-\mathrm{Y}$ displacements, from which a direction and magnitude were calculated: the direction is the estimated direction, and the magnitude can be thought of as the strength of evidence for this estimate. For the OLE, RGC responses, $\overrightarrow{\mathbf{r}}$, were quantified by the number of spikes within a set number of sequential frames (bins) as indicated by the temporal integration time; $\overrightarrow{\mathbf{r}}$ includes an added constant that allows for a default (offset) direction weight. For the OQE, $\overrightarrow{\mathbf{r}}$ includes not only responses of individual cells but also the cross products of all possible cell pairs (Shamir and Sompolinsky, 2004); $d t$ allows a time delay between response and the stimulus and was optimized to minimize the mean squared error between the direction estimate and the true direction. The weights, $\mathbf{W}$, were determined during a separate training set using MATLAB's backslash operation: 


$$
\mathbf{W}=\overrightarrow{\mathbf{r}}(t+d t) \backslash \mathbf{S}
$$

$\mathbf{S}$ is the cartesian coordinates of the stimulus direction. MATLAB's backslash operation returns a least-squares solution to a system of linear equations. Training sets for the dynamic motion stimulus consisted of the first $3 / 4$ time points, and it was tested on the last $1 / 4$ time points. Training and testing on fully separated data blocks prevent the decoder from leveraging the peri-stimulus time histogram (PSTH) autocorrelation to improve its test error. For the static motion stimulus, the OLE and OQE were trained on five images and tested on a hold-out image. Training and testing were redone for each image and errors averaged across all images.

To break correlation structures between cells, binned responses within the test data for the static direction stimulus were circularly shifted by a random amount independently for each cell. This manipulation maintained the direction selectivity of the response averages but broke correlations between cells.

\section{ooDSGC simulation}

A simulation of ooDSGC receptive fields was used to test the observed results in larger and more complete mosaics than available from the measured data. The model consisted of four independent mosaics of modeled receptive fields responding to a moving image. As in the analysis of the measured data, the responses of the modeled neurons were combined to estimate the direction of image motion.

The response, $r(t)$, of an individual model neuron was produced by computing:

$$
r(t)=N\left(F_{\phi p}(x, y, t) * s(x, y, t)\right)
$$

where $F_{\phi p}$, is the linear filter given by

$$
F_{\phi p}(x, y, t)=\cos ((\mathrm{x} * \cos \phi+y * \sin \phi+\psi(t)) * 360 / \lambda) * m v g(\overrightarrow{\boldsymbol{\mu}}, \sigma) .
$$

The parameters of $F_{\phi p}$ are the preferred direction, $\phi$, and the spatial position, $\overrightarrow{\boldsymbol{\mu}}$, of the filter. Linear filters were constructed from a space-time Gabor function using of a set of 10 sinewaves with 150 pixel wavelengths, $\lambda$, that were phase shifted, $\psi(t)$, by 8 pix/frame, and multiplied by a multivariate Gaussian ( $\mathrm{mvg}$ ) with a SD, $\sigma$, of 17 pixels, centered at $\overrightarrow{\boldsymbol{\mu}}$ (the $\mathrm{x}-\mathrm{y}$ position of the receptive field center) and cut off in a square 75 pixel window. The stimulus was $s(x, y, t)$; indicates convolution; and $N$ is a nonlinear function. The position, $\overrightarrow{\boldsymbol{\mu}}$, of each filter, $F_{\phi p}$, was determined by an exclusion zone algorithm for generating 2-dimensional spatial mosaics (Galli-Resta et al., 1999). $N$ was a rectified linear function symmetric about zero (Fig. 5A), allowing responses to be ON-OFF and controlling response sparsity by changing the length of the central (flat) segment of the nonlinear function. The threshold of the nonlinearity was adjusted to provide a similar fraction of spikes as that measured in the ooDSGCs population (90-95\% of the bins had no activity). The density of the simulated mosaics was 30 cells $/ \mathrm{mm}^{2}$ based on reported values in ooDSGCs in rabbit (Vaney, 1994). The population response from these stimulated responses, $\overrightarrow{\mathbf{r}}$, was decoded using the same procedures applied to the data (see Eq. 1).

To understand how high-frequency noise constrained decoding error we added Gaussian white noise with a variance equal to the signal variance. Decoding was performed on the noisy signals without manipulation or after averaging from 10 -frame bins.

\section{Spatial-temporal trade-off simulation}

A simulation of signal estimation was used to understand the tradeoffs inherent in separating signal from noise through spatial and temporal integration. The signal, $\mathrm{S}$, was constructed by convolving spatial and temporal Gaussian filters with white noise, creating a signal dominated by low spatial and temporal frequencies. Unfiltered white noise was then added to $\mathrm{S}$. Then the noisy signal was filtered by convolving spatial and temporal Gaussian filters defined by their SDs, $\sigma$. The mean squared error was calculated between the filtered output and $\mathrm{S}$.

\section{Results}

Visually driven responses of RGCs were measured ex vivo using a MEA (Yao et al., 2018). Responses to drifting gratings distinguished ooDSGCs and oDSGCs from other RGCs over the MEA (see Materials and Methods). To measure the responses of DSGCs to dynamic global motion, a natural scene from the van Hateren image database (van Hateren and van der Schaaf, 1998) was dynamically moved over the retina (Fig. 1A). This paradigm drove the responses of dozens of identified and simultaneously recorded ooDSGCs and oDSGCs.

\section{Individual ooDSGCs encode direction via integration of dynamic global motion}

We begin by focusing on ooDSGCs and analyzing the relationship between their spiking and the dynamic global motion of a natural scene. We randomly and iteratively translated a natural scene on the retina while recording ooDSGC spikes (see Materials and Methods). The $\mathrm{X}$ and $\mathrm{Y}$ positions of the image were shifted in each frame of the video display by $\Delta \mathrm{X}(\mathrm{t})$ and $\Delta \mathrm{Y}$ (t). Image displacements had a Gaussian distribution and were uncorrelated or "white" at temporal frequencies above $0.5 \mathrm{~Hz}$ (Fig. 1A; see Materials and Methods). The image displacement distribution had zero mean with a SD $\sim 20 \mu \mathrm{m} /$ frame $\left(\sim 25^{\circ} / \mathrm{s}\right)$. This value was chosen to maximize responses from ooDSGCs and fall within the range of eye movement velocities in freely moving rats (Wallace et al., 2013) and retinal image motion in rabbits (Van der Steen and Collewijn, 1984). We calculated the correlation between $\Delta \mathrm{X}$ and $\Delta \mathrm{Y}$ values and spike times, yielding a spike-triggered average (STA) of the displacements in X and $\mathrm{Y}$ for each cell (Fig. 1B; Borghuis et al., 2003; Perge et al., 2005; Kühn and Gollisch, 2019). Translating these Cartesian to polar coordinates facilitated visualizing the STA directions of all ooDSGCs simultaneously (Fig. 1C). Approximately $500 \mathrm{~ms}$ preceding a spike, the average motion direction fluctuated randomly for every ooDSGC (Fig. 1Ci). However, between 300 and $100 \mathrm{~ms}$ preceding a spike, the motion direction coalesced to one of four cardinal directions. These results indicate that each ooDSGC encodes global motion along one of four directions and that spiking depends on the motion direction over $\sim 200$-ms temporal window, with $\sim 100$-ms latency to spiking (Fig. 1C).

There are two possible strategies by which ooDSGCs may encode this motion. First, ooDSGCs may simply integrate motion signals over a temporal window. Alternatively, they may signal a change in direction by differentiating the motion trajectory. Differentiation is a common computation performed by the receptive fields of most RGCs (e.g., center-surround antagonism; Kuffler, 1953; Perge et al., 2005; Schwartz et al., 2007). From a linear systems perspective, pure integration requires a monophasic dependence on motion trajectories preceding spikes, while differentiation (in the case of direction changes $>90^{\circ}$ ) requires a biphasic dependence on motion trajectories. Every ooDSGC exhibited a monophasic direction STA (peak/trough ratios at $14.2 \pm 2.7$; Fig. $1 B$ ), with a mean half width of $111 \pm$ $2 \mathrm{~ms}$ (Fig. 1Cii). Thus, ooDSGCs encoding appears more related to the integration of direction for global motion stimuli within relatively short time windows preceding their spikes; they do not appear to explicitly encode changes (differentiation) in the motion direction. Note, the distribution of velocities preceding spikes is Gaussian and largely samples a response range for the 
ooDSGCs that is approximately linear with half-wave rectification (Fig. 1Cii, inset). Below, we explore the implications of needing to decode ooDSGC signals that are updated continuously over short $(200 \mathrm{~ms})$ time windows.

\section{Individual ooDSGCs generate sparse and broadly tuned responses to global motion}

The analyses above reveal the average motion kinetics and directions that precede ooDSGC spiking for global motion in a natural scene. However, the fidelity of encoding, and the accuracy of decoding, will depend strongly on the spiking dynamics elicited by these stimuli. Spiking was infrequent in ooDSGCs to natural scene global motion (Fig. 1D,E), consistent with other measures of RGC activity during natural movie presentations (Koch et al., 2006). For the global motion stimulus, firing rates ranged from 0.8 to $8.5 \mathrm{~Hz}$, with one or more spikes occurring in a single neuron on $<8 \%$ of the video frames ( $40-\mathrm{Hz}$ frame rate). This result was replicated with several different images and natural movies from cameras that were head mounted to animals (Fig. 1Eii; see Materials and Methods), demonstrating that global motion in natural scenes typically evokes sparse responses across ooDSGCs.

One question that arises is whether or not these stimuli were reliably driving spikes in ooDSGCs, given the low spike rates. Repeated presentations of the same stimulus produced stereotyped ooDSGC responses (Fig. 1Fi), indicating that the response sparsity is not simply a result of presenting a stimulus that is incapable of evoking a response. Instead, these stimuli generated sparse responses that were reliable from trial to trial, within each response frame, the spike count mean was approximately equal to the variance (mean fano factor \pm SEM $=1.1 \pm 0.05$ ). However, the motion direction $\sim 200 \mathrm{~ms}$ preceding individual spikes was highly variable (Fig. $1 G$ ). To quantify the variability in motion direction preceding spikes, we calculated the difference between the direction of motion preceding each spike and the STA direction (evaluated at the peak of the STA magnitude). This distribution is broad and on average the direction preceding a spike differs from the mean (preferred) direction by $\pm 70^{\circ}$ (Fig. $1 G$, gray distribution and black dashed line). Even when the displacement $(\Delta \mathrm{X}$ and $\Delta \mathrm{Y})$ is filtered by the STA, the distribution of directions preceding spikes remains relatively broad (Fig. $1 G$, red distribution): on average the direction preceding a spike differs from the mean (preferred) direction by $\pm 53^{\circ}$ (Fig. $1 G$, red dashed line). This variability will limit decoding performance, because the presence of a spike poorly constrains the preceding motion direction.

Variability in the prespike direction likely reflects several sources including: the tuning width of the ooDSGC, different direction trajectories across video frames filling the ooDSGC integration time, and aperture effects that allow local orientation to influence apparent direction within a receptive field (McDermott et al., 2001; Sung et al., 2009; Kane et al., 2011). Irrespective of the source, the stimulus variability preceding ooDSGC spiking combined with infrequent spiking, will limit the accuracy with which direction of global motion can be decoded from ooDSGC populations. Below we assess whether the response properties described above are unique to ooDSGCs, or whether these observations also apply to oDSGCs.

\section{oDSGCs respond similarly to ooDSGCs}

Previous work has suggested that signaling self-motion is performed by oDSGCs while ooDSGCs signal local object motion (Vaney et al., 2001). Thus, we compared the responses of oDSGCs to ooDSGCs to see whether they exhibited distinct response properties to global motion in natural scenes. First, oDSGCs showed similar monophasic temporal integration to ooDSGCs (Fig. 1B, inset). Second, oDSGCs showed similar response sparsity to the same global motion stimuli (Fig. 1Ei). Indeed, all recorded RGCs exhibited similar response sparsity (Fig. 1Ei). oDSGCs also exhibit similarly reliable responses to repeated presentations of the same global motion sequence for a natural scene (Fig. 1Fii) and similar direction variability preceding a spike as ooDSGCs (Fig. $1 G$, inset). Thus, we did not observe clear differences in the response statistics or encoding properties between oDSGCs and ooDSGCs to global motion of a natural image.

The analyses below leverage the simultaneously recorded populations of ooDSGCs to test the ability to decode the direction of global motion from those populations and analyzes the factors limiting the accuracy of that decoding. We focused on ooDSGCs because our MEA measurements contain much larger populations of these cells than oDSGCs.

\section{Quartets of ooDSGCs exhibit limited accuracy in signaling the direction of global motion}

To begin to understand how the response properties of ooDSGC impact the decoding of global motion, we applied an optimal linear estimator (OLE) to the responses from quartets of simultaneously recorded ooDSGCs. In brief, an OLE assigns a set of weights to each cell which, when scaled by the response of that cell and summed across cells, will minimize the mean squared error of the prediction (Fig. 2A; see Materials and Methods). Each quartet consisted of ooDSGCs with different preferred directions and cells within $200 \mu \mathrm{m}$ of one another (Fig. $2 B$ ). We begin with quartets of ooDSGCs because they form an elementary unit of a population code. Specifically, spikes from one ooDSGC poorly constrain motion direction, because of the broad direction range that can precede a spike. However, spikes distributed across a quartet of ooDSGCs can, in principle, be used to more accurately decode motion direction (Georgopoulos et al., 1986). We begin with an OLE because it is a simple decoder that performs nearly optimally on ooDSGC population responses and can be simply implemented by downstream neurons (Salinas and Abbott, 1994; Fiscella et al., 2015).

The first question we address with this approach is the following: "how accurately can global motion in a natural scene be decoded from the responses produced by a local quartet of ooDSGCs?" The answer is likely to depend on the duration over which the decoder integrates signals from the ooDSGCs, and the dynamics of the motion (e.g., how frequently the velocity changes). First, we examined the dependence on integration time. When integration time is short, the decoder is forced to estimate direction from responses produced within single video frames ( $\sim 25 \mathrm{~ms})$. This yielded low-accuracy estimates of motion direction; the median expected error was $\sim 80^{\circ}$ (Fig. $2 C, D$ ). Note this analysis allows for a latency between the stimulus direction and ooDSGC responses (see Materials and Methods). The median error is reported throughout and provides the minimum error in decoding $50 \%$ of the time bins, an appropriate quantity when decoding continuously. For comparison, chance performance in direction estimation would be $90^{\circ}$, and perfect performance would be $0^{\circ}$. A major contributor to this high uncertainty in motion direction is that within $\sim 25 \mathrm{~ms}$, the most frequent output from the quartet of ooDSGCs is zero spikes (Fig. 2E). When there are no spikes, the decoder assigns a default constant, effectively guessing at the direction of motion. It is worth noting that in a stimulus regime with constantly changing direction, this 
A

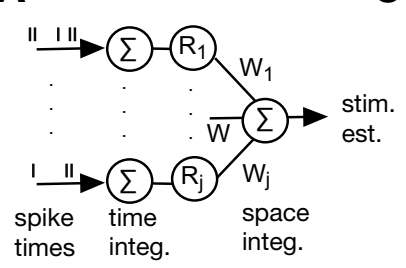

B



D



C

E


F

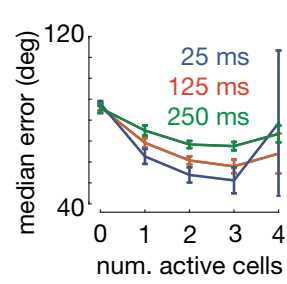

G



Figure 2. 0oDSGC quartets are limited in their decoding accuracy by response sparsity and dynamic motion. $\boldsymbol{A}$, Schematic of the decoder, the OLE. $\boldsymbol{B}$, Examples of four ooDSGC quartets. Relative location of each cell is marked from position on array and circles drawn, $300 \mu \mathrm{m}$ in diameter, provide a scale bar near the size of ooDSGC dendritic fields. Inset shows direction preferences in visual coordinates (S: superior; P: posterior). C, top, Spike response from an example quartet during a dynamic global motion stimulus (spike times are uniformly shifted for optimal decoding). Middle, Decoder provides estimate of direction for each frame change using two different spike integration times. Bottom, Acute difference between OLE and actual direction for each integration time. $\boldsymbol{D}$, Median error over all decoded time points as a function of integration time from one retina $(N=15$ quartets). $\boldsymbol{E}$, Fraction of bins with non-zero spikes in $0-4$ cells in each quartet (same retina as in $\boldsymbol{D}$ ). $\boldsymbol{F}$, Median error as a function of number of cells in quartet with non-zero spikes (same retina as in $\boldsymbol{D}$ ). $\mathbf{G}$, Median error for all decoded time points as a function of integration time when decoding drifting image with direction and speed held constant (see Materials and Methods). Separately recorded retina from panels $\boldsymbol{B}-\boldsymbol{F}$ (seven quartets). Error bars $(D-G)$ show SD across quartets.

default is no worse than assuming the direction that was last decoded when spikes occurred.

To test that the high error at short integration times results from the sparsity of the population response, we analyzed the frequency with which a given number of ooDSGCs responded within a quartet. For short integration times there is a high probability of zero spikes from any ooDSGC in the quartet (Fig. $2 E$ ). Furthermore, decoding error depended on the number of cells responding within a given integration window, the error decreased sublinearly for increasing cell numbers (Fig. 2F). Errors were high when four cells were responding in the same bin, which results from cancelation of oppositely tuned neurons.

One path toward improving decoding performance is for the decoder to integrate over longer time windows. This would allow for a larger fraction of decoded epochs to contain at least one spike from the quartet of ooDSGCs. However, increasing the integration time to $125 \mathrm{~ms}$ (five stimulus frames) only modestly decreased the error of direction estimates to $\sim 74^{\circ}$. Furthermore, for longer integration times, average direction error increased (Fig. 2D). Thus, decoding global motion from local quartets of ooDSGCs exhibits limited accuracy.

The increase in decoding error at longer integration times is likely a result of the dynamic stimulus, which frequently changes directions. Thus, integrating for longer periods of time incurs a cost: the inability to decode frequent changes in direction. To test this hypothesis, we switched from decoding an image that changed direction and speed dynamically to a drifting natural image that moved in a constant direction and speed (see Materials and Methods). As hypothesized, images moved in a static direction show only increases in accuracy with increasing integration time (Fig. 2G), as the decoder was afforded the opportunity of accumulating spikes over long periods of time without a change in direction. Using a 2-s integration window to decode the direction of a drifting natural image reduced the average error down to $\sim 20^{\circ}$ when decoding from a quartet of ooDSGCs.

The analyses above show that quartets of local ooDSGC provide little information about global motion direction in a natural scene at short time scales. Their limited decoding accuracy is largely due to the sparse (infrequent) spiking generated by the stimulus. Furthermore, decoding is limited to short integration times when motion is dynamic because integrating over longer time windows fails to track changes in motion direction. This is at least partly a consequence of ooDSGCs integrating, instead of differentiating, motion (Fig. 1B). If decoding accuracy is limited by the sparsity of the population response, do larger populations of ooDSGCs allow for more accurate decoding of dynamic motion at short integration times?

\section{Large ooDSGC populations can encode direction continuously over short time scales}

To begin to test the effect of ooDSGC population size on decoding global motion, we decoded the direction of dynamic global motion using the responses of all ooDSGCs measured in an experiment (Fig. 3A). While these populations are not complete, due to imperfect sampling of RGCs over the MEA, this analysis permitted data-based decoding on 43-48 ooDSGCs in individual experiments. Furthermore, the population spanned lengths of $\sim 750 \mu \mathrm{m}\left(25^{\circ}\right.$ of visual arc) on the retina.

Using larger populations of ooDSGCs increases the frequency with which one or more cells spike for a given integration time, relative to quartets. This effectively decreases the sparsity of the population response to which the decoder has access. As a result, the median error from decoding these larger population 
A

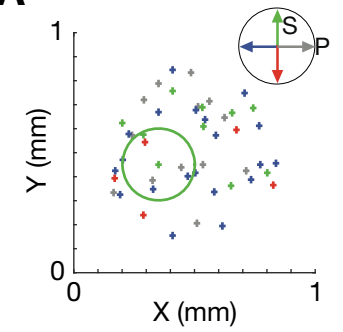

C



$\mathbf{F}$

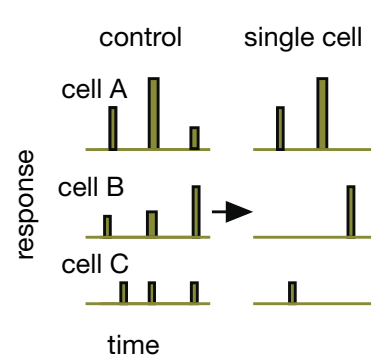

B

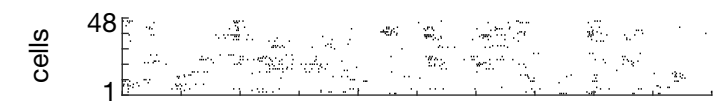





25 ms est.

250 ms est.

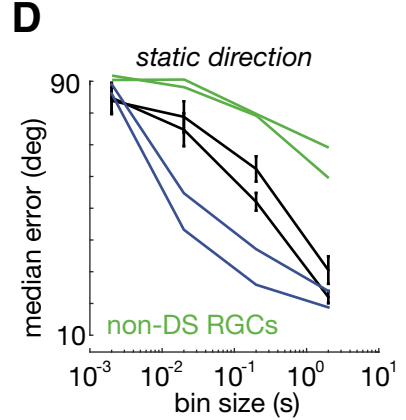

G

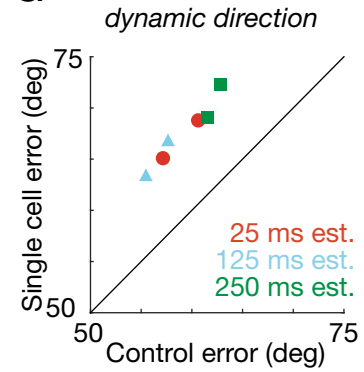

E

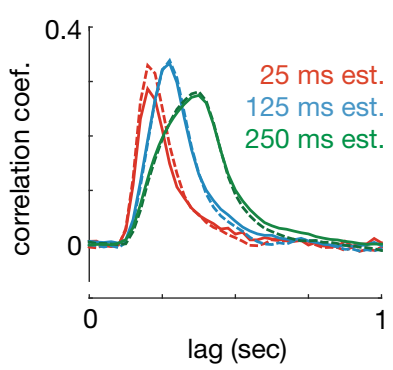

H



Figure 3. Larger populations of ooDSGCs improve decoding accuracy with shorter integration times. $A$, Location on array of all ooDSGCS recorded in single retina. Circle provides estimate of the size of receptive field and colors indicate preferred direction. Inset shows direction preferences in visual coordinates (S: superior; P: posterior). B, top, Spike response from recorded ooDSGC population during dynamic global motion stimulus. Middle, Decoder provides estimate of direction for each frame change using two different spike integration times. Bottom, Acute difference between OLE and actual direction for each integration time. C, Median error for all decoded time points as a function of integration time. For both the quartets (as in Fig. 2D) recorded in two different retinas and the entire population for two different retinas. No error bars are provided for the estimate across the entire population. $\boldsymbol{D}$, As in panel $\mathbf{C}$ using an image with a constant direction (see Materials and Methods) with green showing performance of non-DS RGCs for comparison. $\boldsymbol{E}$, Cross correlation between stimulus image displacement estimate and actual stimulus ( $\Delta \mathrm{X}=$ solid or $\Delta \mathrm{Y}=$ dashed line). $\boldsymbol{F}$, Schematic showing "single cell" manipulation of input to OLE. $\boldsymbol{G}$, Median error using control and single cell input to OLE. Results from two retinas are shown at three different spike integration times. $\boldsymbol{H}$, Same as panel $\mathbf{G}$ using image with direction held constant.

responses was significantly smaller than decoding ooDSGC quartets, particularly for short integration times (Fig. 3B,C). For example, at $\sim 25 \mathrm{~ms}$ (a single video frame), decoding error was reduced to $55-60^{\circ}$ for a population of 48 ooDSGCs, down from $80^{\circ}$ for a quartet. It is notable that decoding direction from a population on a single frame was so accurate, given that a single frame is much briefer than the integration time of the STA (Fig. $1 B)$.

Similar to the results from ooDSGC quartets, increasing the integration time also caused an increase in median errors for larger ooDSGC populations (Fig. 3B,C). This increase is because the global motion is dynamic, causing the decoder to estimate a single direction of motion from responses that are produced by multiple directions. When the direction of the stimulus was constant, longer integration times resulted in a monotonic decrease in error for large populations of ooDSGCs (Fig. 3D, blue). For example, at 2-s integration, decoding error fell to $\sim 20^{\circ}$ with a population of 48 ooDSGCs. For comparison, an OLE used to decode motion direction from equivalently-sized populations (43 and 48) of non-DS RGCs (recorded simultaneously) performed much worse than ooDSGC populations or even ooDSGC quartets (Fig. 3D). This demonstrates that despite the somewhat low precision in estimating direction of global motion at short timescales, DSGCs provide much more information than other RGCs. In summary, larger ooDSGC populations allow for more accurate decoding of global motion in natural scenes within briefer integration times. However, long integration times limit decoding performance when global motion changes rapidly.

Thus far, we have shown that long stimulus integration impairs the ability of ooDSGC populations to accurately estimate dynamic motion. Long stimulus integration has an additional cost, which is to delay the time at which direction estimates are most accurate relative to the stimulus. To measure this delay, we computed the cross-correlation between the actual and estimated image displacements (in $\Delta \mathrm{X}$ and $\Delta \mathrm{Y}$ ). The cross-correlation between these values was significantly delayed and broader at longer integration times (Fig. 3E). Thus, integration over short timescales allows downstream circuits to decode more rapidly, thereby following more frequent changes in direction. This is only achievable with large populations of ooDSGCs because quartets perform marginally better than chance within the same integration times. 
Increasing the population size could improve decoding in two different ways: (1) by increasing the number of time points with single responsive cells; and/or (2) increasing the number of time bins with multiple responsive cells. To measure the extent to which the error depended on a simultaneous multi-cell response, the OLE was trained on the full response set and tested on either the full response set, or on a modified response set in which only a single cell response (the largest response) at each time bin was provided to the decoder (Fig. 3F). If direction decoding is entirely mediated by single cells, then there should be no difference between using the full and modified response sets. There was a substantial increase in the error when decoding on the modified response set in both the dynamic (Fig. $3 G$ ) and static (Fig. $3 H$ ) direction stimuli. Thus, the decoding accuracy in larger populations relies on simultaneous activity from multiple ooDSGCs.

The simultaneous activity between ooDSGCs that underlies a population response could arise purely through independent responses across ooDSGCs or through correlated subsets of ooDSGCs. In the next section, we examine the extent to which the accuracy of rapid decoding in large ooDSGCs populations relies on response correlations within the population.

\section{Rapid-global motion direction is encoded by large populations of independent ooDSGCs}

Natural scenes have local intensity correlations that result in correlated activity between nearby RGCs (Simoncelli and Olshausen, 2001; Pitkow and Meister, 2012). Recent work has indicated that such response correlations promote robust decoding by maintaining the relative activity between ooDSGCs with different preferred directions (Franke et al., 2016; Zylberberg et al., 2016). To what extent are response correlations important to maintaining the accuracy of rapid decoding of global motion from large ooDSGC populations?

To understand how the correlation structure contributes to decoding accuracy, we measured and manipulated response correlations across the ooDSGC populations. In this section, we focused entirely on the static direction stimulus, which permitted manipulations that would be impossible across a dynamic direction stimulus.

First, we examined the correlation structure in the population by mapping the pairwise correlation coefficients as a function of (1) distance between pairs, (2) relative preferred direction, and (3) integration time (Fig. 4Ai-Aiii). The correlation coefficients were calculated within a trial and averaged across all trials and directions. Thus, the response correlations reported here include signal and noise correlations and measure the tendency of cells to respond to the same image structure. The correlation coefficient between pairs of ooDSGCs increases with the integration time used to calculate the responses, as previously noted (Cohen and Kohn, 2011), short integration times diminish correlations (Fig. $4 A$, note axes scale). Thus, over short integration times, correlations are small, suggesting they may not influence decoding accuracy to the extent observed in previous studies that considered longer integration times (Franke et al., 2016; Zylberberg et al., 2016).

However, for a given integration time, the correlation is higher for cells that are spatially closer and modulated less
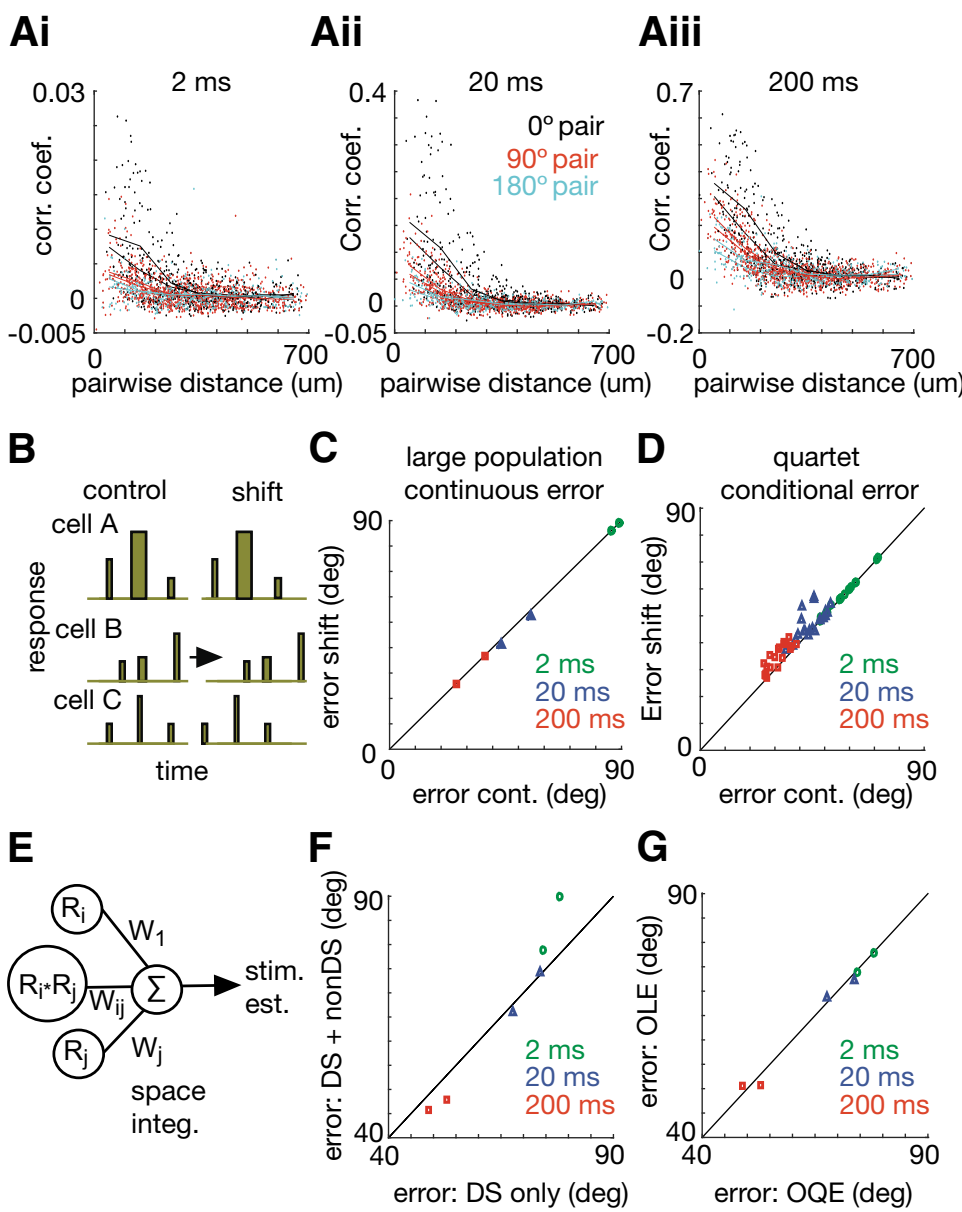

Figure 4. Correlation coefficients are small on short time scales and have little impact on decoding accuracy. $A$, Correlation coefficient between pairs of ooDSGGs as a function of the distance between pairs. All possible pairs within a retina are plotted for two different retinas (points) and the averages within a binned distance are drifting grating. Panels $\boldsymbol{i}$-iii present the same data but calculated using three different integration times. $\boldsymbol{B}$, (he input to the OLE. C, Median error using control and shifted tude direction estimates. $E$. Schematic illustrating decoding with OOE $F$. Decoding error of small local population of OODSGCS and non-DS cell responses using an OQE. G, Decoding error of small local population of OODSGCS only using an OQE and OLE.

prominently by their relative preferred directions (Fig. 4Ai; Pitkow and Meister, 2012). This reflects the increased tendency of nearby cells to respond to the same part of the image as the dominant determinant of correlation structure. This led us to ask whether the higher correlations in nearby cells are important in maintaining the accuracy of decoding from large ooDSGC populations over short time scales. In other words, are responses to global motion encoded by many small-local populations of correlated cells?

To test how decoding error depends on the correlations between ooDSGCs, the OLE (trained on an unmodified response set) was tested on either an unmodified (cross-validated, control) response set or a decorrelated (shifted) response set, in which the response bins were shifted in time during a drifting image (Fig. $4 B$ ). Shifting responses in time independently across ooDSGCs eliminates correlations due to local contrast fluctuations in the stimulus and noise correlations introduced by retinal circuits. However, this manipulation maintains correlations due to the direction of motion. Thus, shifting responses in time undermines the population response structure caused by the particular spatial 

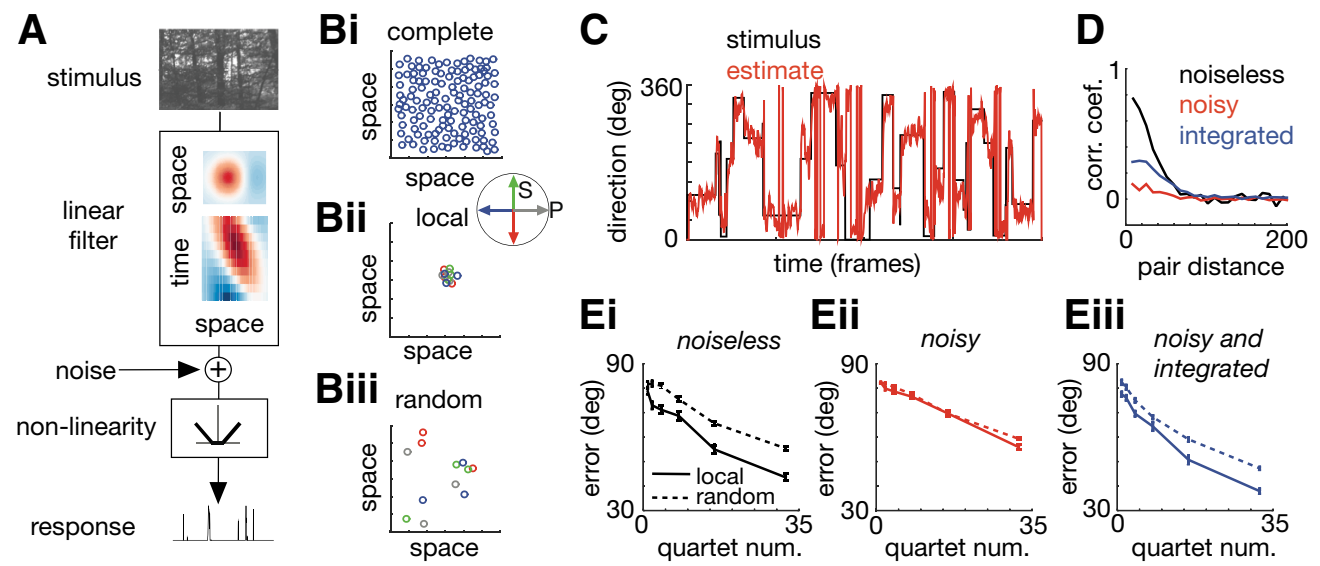

Figure 5. Model of ooDSGC populations demonstrates how natural images, integration time, and noise influence correlations and decoding. $\boldsymbol{A}$, Schematic of a single modeled DS-unit. $\boldsymbol{B}$, Example of receptive field positions subsampled from complete DS-unit mosaics (Bi, showing one of four mosaics) when decoding three spatially local (Bii) or random (Biii) quartets. Circles illustrate 1 SD of receptive field size. Inset shows direction preferences in visual coordinates (S: superior; P: posterior). $C$, Example of image direction and decoded direction estimate at each point in time. $\boldsymbol{D}$, Average pairwise correlation coefficient within a large population of modeled DS-units as a function of distance between pairs. $\boldsymbol{E}$, Decoding error versus size of DS-unit population in either a noiseless model (E⿱一𫝀)), a noisy model (Eii), or a noisy model with temporal integration (Eiii).

locations of the cells, and is similar to selecting populations of ooDSGCs randomly in space. Across a range of integration times, the "shifted" response sets showed little change in continuous decoding error (Fig. 4C). This result suggests that decoding of direction from large ooDSGC populations does not depend on correlations, even when those correlations are relatively large.

To further investigate the role of correlations in decoding ooDSGC population responses, we again decoded the direction of natural images moving in a constant direction and speed. Instead of shifting ooDSGC responses in time, we shuffled ooDSGC responses across repeated presentation of the same stimulus. This manipulation preserves spiking correlations that are stimulus induced; e.g., those caused by cells observing the same motion direction and those caused by local contrast structure in the image. However, this manipulation specifically eliminates noise correlations. The results were very similar to those obtained by time shifting the responses (results differed by $<2 \%$ at 2-, 20-, and 200-ms decoding windows from time shifting responses, comparison includes two ooDSGC populations of 43 and 47 ooDSGCs from two retinas).

These result differ substantially from previous findings where trial-to-trial noise correlations alone were shown to significantly decrease decoding error by maintaining orthogonality between signal and noise (Franke et al., 2016; Zylberberg et al., 2016). However, there are three critical differences: First, we are decoding ooDSGC responses over shorter time windows, which will tend to reduce the amount of correlations and their impact on decoding. Second, we are decoding global motion instead of local motion, which reduces the number of spikes and the amount of correlated spiking. Third, previous studies focused almost exclusively on DSGCs with large amounts of receptive field overlap to maximize the amount of signal and noise correlations. This predicts that if we focused on the strongest responses (those with the most spikes), decoded over longer time windows, and only analyzed cells with large RF overlap, we should start to observe an effect of removing correlations on direction decoding.

To test this prediction, we focused on decoding quartets with high RF overlap during time periods when they were strongly responding. We used the OLE magnitude to select the time bins in which the ooDSGCs population was responding most strongly (Fig. 2A). The OLE magnitude will be highest when multiple cells, with similar tuning are responding most strongly. We assess the median error during the top 10\% magnitude responses and term this the "conditional error" (because it is conditioned on the OLE magnitude being high). As predicted, the conditional error in quartets began to be sensitive to correlation structure at long integration times (Fig. 4D). Specifically, shifting the response bins in time to a constantly drifting image increased the error at long integration times but not at short integration times (Fig. 4D). Similar results were obtained when shuffling responses across repeated presentation of the stimulus, to specifically eliminate noise correlations (data not shown). These results are smaller than in previous studies (Franke et al., 2016; Zylberberg et al., 2016), likely because ooDSGC produce (even during the strongest responses) fewer spikes to global motion in a natural image than to a high contrast circle or bar moving on a uniform gray background. Thus, the impact of correlations on decoding depends critically on the integration time for decoding and the nature of the stimulus-response relationship, correlations being important when decoding large responses integrated over long time windows. We later extend these results to large modeled DS populations with complete mosaics (Fig. 5).

\section{Non-DS cells do not improve direction decoding over short integration times}

The analyses above indicate that correlations between ooDSGCs are not useful to account for local image intensity when decoding the direction of motion in short integration times. However, local image intensity influences the spike rates of non-DS RGCs, as well as DSGCs. Potentially, non-DS RGCs that share substantial receptive field overlap with ooDSGCs could be used to help decode the direction of motion by discounting local image intensity. Indeed, correlations between tuned and untuned neurons have been shown to improve decoding from other neural populations (Leavitt et al., 2017; Zylberberg, 2018).

To assay whether non-DS RGCs can help decode the direction of motion, we decoded using both identified ooDSGCs and non-DS cells. Using local groups with substantial receptive field overlap ( $\sim 30$ neurons), the direction of motion was first decoded using an OLE. There was not a significant difference between decoding performance with or without non-DS cells (data not shown). This result is expected, because an OLE, while sensitive to correlation structure, does not explicitly use correlated activity 
to decode (Schneidman et al., 2003; Shamir and Sompolinsky, 2004). Consistent with this result, only using non-DS RGCs to decode motion direction with an OLE yielded very poor decoding performance (Fig. $3 D$ ). Alternatively, an $\mathrm{OQE}$ explicitly uses correlations between neurons to decode by weighting the synchronous activity between neurons to estimate the direction of motion (Shamir and Sompolinsky, 2004; Fig. 4E; see Materials and Methods). However, like the OLE, the OQE accuracy was similar when decoding from DS only versus DS and non-DS populations over short integration times (Fig. $4 F$ ). There was a small decrease in error when including non-DS cells and decoding with longer integration times (Fig. $4 F$ ). Indeed, the $\mathrm{OQE}$ and OLE using DS cells alone provided similar accuracy (Fig. 4G), indicating little benefit from this form of nonlinear decoding in these conditions. Finally, the OLE and OQE also performed similarly when using only non-DS RGCs, with the OQE exhibiting $4^{\circ}$ improvement (median over 2-, 20-, 200-, and 2000-ms time bins) in decoding performance over the OLE (data not shown). Thus, decoding of the direction of motion continuously with short integration bins is effectively performed by a linear decoder that integrates signals from a large tuned population with little benefit from correlated activity between tuned or untuned neurons. Note, we are not saying that other classes of decoders that are more explicitly constructed to decode motion from untuned neurons could not be useful for bolstering signals from DSGCs. For example, Reichardt detectors, which introduce a temporal delay between the joint activity of RGCs can be used to decode motion from non-DS RGC signals (Frechette et al., 2005).

\section{Noise and temporal integration dictate spatial decoding constraints in model}

The analyses above indicate that large populations of nearly independent ooDSGCs can be leveraged to rapidly decode the direction of motion, while increasing temporal integration increases the importance of spatial correlation structure for accurate decoding. Ostensibly, the relationship between temporal integration and sensitivity to local correlations could be explained by the presence of high temporal frequency noise in ooDSGC responses. To better understand how noise and temporal integration influence population decoding we created a model that simulated responses from complete ooDSGC mosaics of various sizes and organizations (see Materials and Methods). In brief, each modeled DS-unit response was generated from a distinct linear-nonlinear model (Fig. 5A) with its position and direction orientation determined by one of four modeled mosaics (Fig. $5 B$ ). The linear filter provides direction tuning and the non-linearity was adjusted to generate on-off responses with sparsity similar to that in the data. The DS-units were stimulated with a moving image used on the retina and the direction of motion was decoded at each time point from the population responses (Fig. 5C). Note, decoding performance for the model (Fig. 5C) was better than for the data (Fig. 2C), because 532 simulated DSunits (133 for each direction) were used in the model, compared with just $\sim 50$ real DSGCs to decode with data. To test the spatial sensitivity of the decoder, populations were decoded either from local subsets (Fig. 5Bii) or from DS-units with randomly chosen spatial locations (Fig. 5Biii).

To begin, the responses of the DS-units were noiseless. In this case, (signal) correlations between nearby DS-units were much higher than that observed in the measured data (Fig. 5D, black curve). These correlations are caused by local image statistics and while they are diminished by the non-linearity that produces the sparse responses, they remain very high between cells with high receptive field overlap. In the absence of noise, decoding error was substantially increased when decoding from DS-units with random spatial locations (Fig. 5Ei) or shuffled responses (data not shown), supporting previous work illustrating the importance of maintaining (signal or noise) correlations in decoding direction (Franke et al., 2016; Zylberberg et al., 2016). Adding independent noise to each DS-unit reduced local (stimulus induced) correlations substantially (Fig. $5 D$, red curve) and greatly reduced the sensitivity of decoder performance on the spatial arrangement of the DS-units (Fig. 5Eii). Finally, temporally integrating the noisy responses partially rescued the correlation structure produced by the natural image (Fig. $5 D$, blue curve) and increased the sensitivity of the decoder to the spatial structure of the correlations (Fig. 5Eiii). This model illustrates that temporal integration influences correlation structure (at least in this example) and that it dictates the decoder's reliance on those correlations. This helps to resolve the discrepancy between this study and previous studies (Franke et al., 2016; Zylberberg et al., 2016), which have highlighted the importance of correlations for decoding DSGC population responses: Here, brief temporal integration was required to decode dynamic global motion, while previous work focused long temporal integration because motion stimuli were local and had a static velocity.

\section{Discussion}

To fully understand neural function, activity must be measured within an appropriate ecological context. While completely natural stimuli are difficult to produce and parameterize (Rust and Movshon, 2005), increasing stimulus complexity toward more natural contexts can help evaluate ideas about neural function derived from using simpler stimuli. In this article, we assayed the potential of populations of ooDSGCs to signal the direction of dynamic global motion of a natural scene. While the stimulus we used falls short of capturing the full complexity of global motion as a mouse moves through the environment, it offers some features beyond drifting gratings or moving spots, while also providing more control over the stimulus than natural movies. For example, this stimulus has naturalistic statistics such as spatial structure that falls off (on average) as the inverse of the spatial frequency, but with high-contrast edges and objects not present in pink noise. Also, the frequency of direction changes can be manipulated to analyze how decoding performance depends on frequent direction changes (Fig. 2D) versus constant velocity motion (Fig. 2G).

Using this stimulus, we describe several novel findings. First, mammalian ooDSGCs integrate global motion signals over $\sim 200 \mathrm{~ms}$ and respond sparsely but reliably (Fig. 1). This sparsity necessitates long integration times to decode accurately using small ooDSGC populations, which precludes decoding frequent changes in motion direction accurately and rapidly (Fig. 2). On the other hand, large populations of ooDSGCs can be used to more accurately and rapidly decode dynamic changes in the direction of global motion (Figs. 3, 5). Together, these findings support the idea that ooDSGCs could contribute to global motion processing (Sabbah et al., 2017). Below, we discuss these findings in the context of previous research.

\section{Functional role of ooDSGCs}

Several lines of evidence have supported the role of ooDSGCs in coding local motion. This is based primarily on three observations: (1) ooDSGCs exhibit diminished spike rates to global 
relative to local motion (so-called surround suppression; Vaney et al., 2001; Chiao and Masland, 2003; Olveczky et al., 2003; Hoggarth et al., 2015; Huang et al., 2019); (2) oDSGCs do not exhibit surround suppression; (3) ooDSGCs have small receptive fields and a high density, which seem unnecessary for signaling global motion (Vaney et al., 2001).

Regarding (1), recent work shows direction selectivity is maintained in global motion and that surround suppression may preferentially attenuate luminance responses (Hoggarth et al., 2015; Im and Fried, 2016). This suggests that the attenuation may emphasize direction information rather than obscuring it. Regarding (2), oDSGCs exhibit similar response structure to ooDSGCs to dynamic global motion (Fig. 1). While the incompleteness of our oDSGC populations prevented an analysis of decoding their responses, the similarity in their encoding properties to ooDSGCs suggests similar decoding performance. Regarding (3), we showed that large dense populations are actually necessary to accurately and rapidly signal dynamic global motion given the sparsity of ooDSGC responses. Finally, a recent study showed that the cardinal axes formed by ooDSGCs align with the axes of self-motion on the retina, suggesting that the population is geared to signal global motion (Sabbah et al., 2017). Thus, we think it is plausible that ooDSGCs play a significant role in signaling global motion.

None of these arguments rule out a role for ooDSGCs to also encode local motion (see below). ooDSGCs clearly respond vigorously to moving spots and bars, which may be reasonable proxies for local motion in nature.

\section{Challenges and constraints to continuously decoding ooDSGC population responses}

Response sparsity and stimulus variability preceding a spike (i.e., broad tuning) challenges a downstream decoder that must (nearly) continuously and accurately estimate a dynamic stimulus. This is a distinct decoding regime from that often investigated; i.e., when constant velocity stimuli produce high firing rates (Theunissen and Miller, 1991; Fiscella et al., 2015; Marre et al., 2015; Kühn and Gollisch, 2019) in a population of narrowly tuned neurons (Jazayeri and Movshon, 2006). Previous studies decoding dynamic motion stimuli from retina have used the optimal linear filter approach (Warland et al., 1997; Marre et al., 2015; Kühn and Gollisch, 2019). This approach temporally integrates spike rates with a fixed filter to provide a continuous optimal linear estimate. Our approach differs from the optimal linear filter approach because we explored a range of integration times. Integrating over or under the optimal temporal range will increase the total mean squared error of the estimate but can decrease the error at specific temporal frequencies. Thus, this approach can highlight tradeoffs inherent in decoding visual information. While previous studies have used long temporal integration windows to improve decoding (Fiscella et al., 2015), we show that such strategies come at a significant cost to temporal resolution for decoding rapidly changing stimuli (Fig. 2D).

To better illustrate the spatial-temporal trade-off in decoding, we constructed a simple model of signal and noise separation (Fig. 6). In this model, noise is separated from signal using a linear filter that integrates over space and time (Fig. 6A). As we observed for decoding ooDSGC population responses, temporal integration can improve signal detection by preferentially attenuating high-frequency noise. This noise reduction strategy is limited though by the presence of a high (temporal) frequency signal, as further integration begins to degrade both signal and noise (Fig. 6Bi). The same problem occurs when integrating
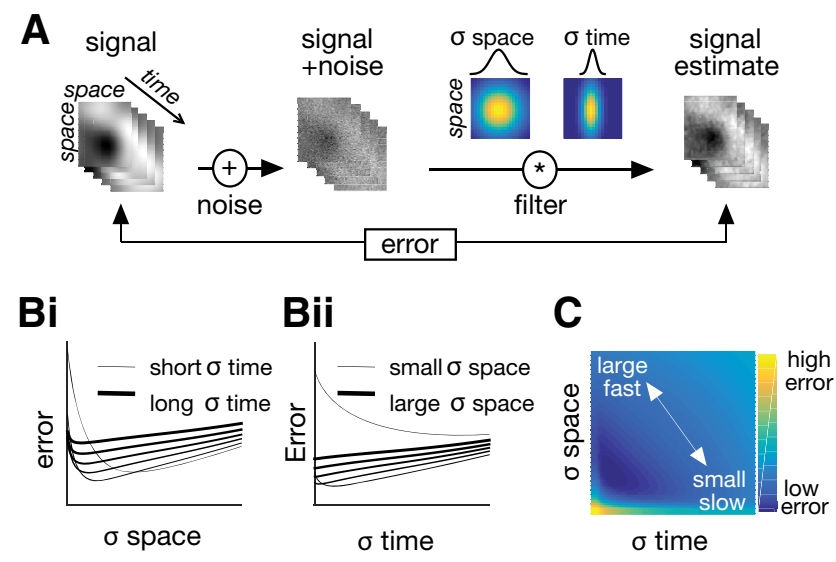

Figure 6. Separating signal and noise using spatial or temporal integration, sparing resolution in the dimension not integrated. $A$, Schematic of simple model illustrating signal corrupted by noise, integration with spatial and temporal linear filters, and estimate comparison. $\boldsymbol{B}$, Error as a function of integrating spatially (Bi) or temporally (Bii) for signals that have been integrated with increasingly long temporal (Bi) or large spatial (Bii) filters. $\boldsymbol{C}$, Error as a function of the temporal and spatial filter size.

spatially (Fig. $6 \mathrm{Bii}$ ). However, the greater the spatial integration, the less temporal integration is needed to improve signal detection, sparing the high-frequency temporal signal. A similar trade-off can be made in the opposite direction, by sacrificing temporal resolution for greater spatial resolution. Thus, in extracting information, a decoder can choose to focus on spatial or temporal resolution, at a cost to the temporal or spatial resolution, respectively (Fig. 6C). Dynamic global motion requires high temporal resolution but minimal spatial resolution: we show that the population response of ooDSGCs permits a regime for accurately decoding this stimulus at short timescales with a simple linear decoder.

\section{Comparison to salamander DSGCs}

This study complements a conceptually similar study recently performed in the salamander retina (Kühn and Gollisch, 2019). In that study, textures were presented and dynamically displaced while recording responses from populations of OFF DSGCs. These OFF DSGCs may be analogous to mammalian oDSGCs, because they exhibit minimal surround suppression and are organized along three directions (Kühn and Gollisch, 2016). Like our study, the authors examined both encoding and decoding performance.

For encoding, salamander OFF DSGCs responded in a manner sensitive to the direction of global motion and integrated motion over $\sim 200 \mathrm{~ms}$, similar to the results presented here. It is interesting to note that the integration was similar despite the difference in species and the substantial differences in the recording temperatures: $21^{\circ} \mathrm{C}$ (salamander) and $34^{\circ} \mathrm{C}$ (mouse).

For decoding, there are several differences between the two studies. First, we used the OLE (and OQE) while the previous study used the "optimal linear filter" approach (see Challenges and constraints to continuously decoding ooDSGC population responses for comment on these approaches; Warland et al., 1997). Second, the previous study quantified decoding performance in terms of Shannon information, while we quantified it in terms of degrees of visual angle. Third, the previous study focused on the synergy available for decoding when cells with different preferred directions were used by the decoder, while we focused on how decoding performance depended on population size, decoding timescale, and correlation structure. Nevertheless, 
a few coherent comparisons can be made. First, both studies indicate that noise correlations play a minimal role in rapidly decoding global motion. Second, both studies show that rapid changes in the direction of global motion are poorly decoded from small populations of DSGCs. For example, extrapolating Kühn and Gollisch (2019), their Figure 2, a population of 20 salamander DSGCs provides little information $(<0.1$ bits in $1 \mathrm{~s}$, or $>80^{\circ}$ expected error) for $>5-\mathrm{Hz}(<200-\mathrm{ms})$ changes in global motion direction. Thus, across species, large populations of DSGCs are required to approach reasonable decoding accuracy for rapid changes in global motion. However, given that the mouse retina has $\sim 8000$ ooDSGCs $\left(\sim 400\right.$ cells $/ \mathrm{mm}^{2} \times \sim 20$ $\mathrm{mm}^{2}$; Rivlin-Etzion et al., 2011; Trenholm et al., 2011) accurate decoding of global motion at short timescales is conceptually achievable.

Our study departs from previous work by analyzing global motion processing in the mouse retina. Furthermore, we explicitly show that (1) the encoding and response statistics of oDSGCs and ooDSGCs are similar for global motion; (2) large populations of ooDSGCs can be used to decode global motion with high temporal resolution; and (3) the nature of ooDSGC population codes depends on the nature of the stimulus and the constraints of the decoding task, such as the necessity of hightemporal versus high-spatial resolution.

\section{The importance of correlation on neural decoding}

The role of correlated activity in neural coding is intensely debated in neuroscience (Schneidman et al., 2003; Latham and Nirenberg, 2005; Averbeck et al., 2006; Shamir, 2014). Mammalian ooDSGCs have provided a useful model system to understand the sources and impact of correlated activity (Amthor et al., 2005; Franke et al., 2016; Zylberberg et al., 2016). Those studies largely pointed to correlations exerting a benefit on DSGC population codes. We show that this result depends on context. When integrating (or decoding) over long timescales, correlation strength can be high and can improve decoding performance (Fig. 4D). However, when integrating (or decoding) over short timescales, correlations are small, even over many cells, and thus decoding performance is independent of the correlations (Fig. 4C,D). We also show that correlations between DSGC and non-DS RGC weakly impacted decoding error over short integration times (Fig. 4G). These observations suggest that shared noise exists at lower temporal frequencies than independent noise. Thus, as demonstrated in a model (Fig. 5), temporal integration diminishes independent noise and strengthens correlations in local populations, shifting the decoder's input from independent to locally correlated populations.

These observations have implications for downstream circuits that process retinal signals. Circuits that decode local motion stimuli can leverage temporal integration to diminish independent noise without sacrificing spatial resolution. This favors a decoder using local correlations, which help maintain a robust population response during a transient stimulus. In contrast, downstream circuits that decode global motion stimuli can pool over large numbers of ooDSGC assuming independence to achieve a nearly continuous readout of motion direction. This suggests distinct decoding regimes - decoders for large, fast visual stimuli relying on independent inputs and decoders for small and slow stimuli using correlated inputs. This may help explain why ooDSGC axons diverge to multiple downstream brain circuits including the lateral geniculate nucleus and superior colliculus (Kay et al., 2011). Future work may reveal that these distinct circuits instantiate these distinct decoding regimes. It is also possible that neuromodulators alter the integration times within a single circuit (Higley et al., 2009), switching between the two decoding regimes dynamically depending on current task demands.

\section{References}

Amthor F, Tootle J, Grzywacz N (2005) Stimulus-dependent correlated firing in directionally selective retinal ganglion cells. Vis Neurosci 22:769-787.

Averbeck B, Latham P, Pouget A (2006) Neural correlations, population coding and computation. Nat Rev Neurosci 7:358-366.

Barlow HB, Hill RM, Levick WR (1964) Retinal ganglion dells responding selectively to direction and speed of image motion in the rabbit. J Physiol 173:377-407.

Betsch B, Einhäuser W, Körding K, König P (2004) The world from a cat's perspective-statistics of natural videos. Biol Cybern 90:41-50.

Borghuis B, Perge J, Vajda I, van Wezel R, van de Grind W, Lankheet M (2003) The motion reverse correlation (MRC) method: a linear systems approach in the motion domain. J Neurosci Methods 123:153-166.

Chiao C, Masland R (2003) Contextual tuning of direction-selective retinal ganglion cells. Nat Neurosci 6:1251-1252.

Cohen MR, Kohn A (2011) Measuring and interpreting neuronal correlations. Nat Neurosci 14:811-819.

Demb J (2007) Cellular mechanisms for direction selectivity in the retina. Neuron 55:179-186.

Devries S, Baylor D (1997) Mosaic arrangement of ganglion cell receptive fields in rabbit retina. J Neurophysiol 78:2048-2060.

Dhande OS, Estevez ME, Quattrochi LE, El-Danaf RN, Nguyen PL, Berson DM, Huberman AD (2013) Genetic dissection of retinal inputs to brainstem nuclei controlling image stabilization. J Neurosci 33:17797-17813.

Elstrott J, Anishchenko A, Greschner M, Sher A, Litke A, Chichilnisky E, Feller M (2008) Direction selectivity in the retina is established independent of visual experience and cholinergic retinal waves. Neuron 58:499506.

Felsen G, Touryan J, Han F, Dan Y (2005) Cortical sensitivity to visual features in natural scenes. PLoS Biol 3:e342.

Field G, Greschner M, Gauthier J, Rangel C, Shlens J, Sher A, Marshak D, Litke A, Chichilnisky E (2009) High-sensitivity rod photoreceptor input to the blue-yellow color opponent pathway in macaque retina. Nat Neurosci 12:1159-1164

Field G, Gauthier J, Sher A, Greschner M, Machado T, Jepson L, Shlens J, Gunning D, Mathieson K, Dabrowski W, Paninski L, Litke A, Chichilnisky E (2010) Functional connectivity in the retina at the resolution of photoreceptors. Nature 467:673-677.

Fiscella M, Franke F, Farrow K, Müller J, Roska B, da Silveira RA, Hierlemann A (2015) Visual coding with a population of direction-selective neurons. J Neurophysiol 114:2485-2499.

Fitzgerald JE, Clark DA (2015) Nonlinear circuits for naturalistic visual motion estimation. Elife 4:e09123.

Franke F, Fiscella M, Sevelev M, Roska B, Hierlemann A, da Silveira RA (2016) Structures of neural correlation and how they favor coding. Neuron 89:409-422.

Frechette E, Sher A, Grivich M, Petrusca D, Litke A, Chichilnisky E (2005) Fidelity of the ensemble code for visual motion in primate retina. J Neurophysiol 94:119-135.

Galli-Resta L, Novelli E, Kryger Z, Jacobs GH, Reese BE (1999) Modelling the mosaic organization of rod and cone photoreceptors with a minimalspacing rule. Eur J Neurosci 11:1461-1469.

Georgopoulos A, Schwartz A, Kettner R (1986) Neuronal population coding of movement direction. Science 233:1416-1419.

Higley MJ, Soler-Llavina GJ, Sabatini BL (2009) Cholinergic modulation of multivesicular release regulates striatal synaptic potency and integration. Nat Neurosci 12:1121-1128.

Hoggarth A, McLaughlin A, Ronellenfitch K, Trenholm S, Vasandani R, Sethuramanujam S, Schwab D, Briggman K, Awatramani G (2015) Specific wiring of distinct amacrine cells in the directionally selective retinal circuit permits independent coding of direction and size. Neuron 86:276-291.

Huang X, Rangel M, Briggman KL, Wei W (2019) Neural mechanisms of contextual modulation in the retinal direction selective circuit. Nat Commun 10:2431. 
Im M, Fried SI (2016) Directionally selective retinal ganglion cells suppress luminance responses during natural viewing. Sci Rep 6:35708.

Jazayeri M, Movshon JA (2006) Optimal representation of sensory information by neural populations. Nat Neurosci 9:690-696.

Kane D, Bex P, Dakin S (2011) Quantifying "the aperture problem" for judgments of motion direction in natural scenes. J Vis 11.

Kay J, De la Huerta I, Kim I, Zhang Y, Yamagata M, Chu M, Meister M, Sanes J (2011) Retinal ganglion cells with distinct directional preferences differ in molecular identity, structure, and central projections. J Neurosci 31:7753-7762.

Koch K, McLean J, Segev R, Freed MA, Berry MJ, Balasubramanian V, Sterling P (2006) How much the eye tells the brain. Curr Biol 16:14281434.

Kuffler S (1953) Discharge patterns and functional organization of mammalian retina. J Neurophysiol 16:37-68.

Kühn NK, Gollisch T (2016) Joint encoding of object motion and motion direction in the salamander retina. J Neurosci 36:12203-12216.

Kühn NK, Gollisch T (2019) Activity correlations between direction-selective retinal ganglion cells synergistically enhance motion decoding from complex visual scenes. Neuron 101:963-976.7.

Latham P, Nirenberg S (2005) Synergy, redundancy, and independence in population codes, revisited. J Neurosci 25:5195-5206.

Leavitt ML, Pieper F, Sachs AJ, Martinez-Trujillo JC (2017) Correlated variability modifies working memory fidelity in primate prefrontal neuronal ensembles. Proc Natl Acad Sci USA 114:E2444-E2503.

Li P, Gauthier J, Schiff M, Sher A, Ahn D, Field G, Greschner M, Callaway E, Litke A, Chichilnisky E (2015) Anatomical identification of extracellularly recorded cells in large-scale multielectrode recordings. J Neurosci 35:4663-4675.

Litke AM, Bezayiff N, Chichilnisky EJ, Cunningham W, Dabrowski W, Grillo AA, Grivich M, Grybos P, Hottowy P, Kachiguine S, Kalmar RS, Mathieson K, Petrusca D, Rahman M, Sher A (2004) What does the eye tell the brain?: development of a system for the large-scale recording of retinal output activity. IEEE Trans Nucl Sci 51:1434-1440.

Marre O, Botella-Soler V, Simmons KD, Mora T, Tkačik G, Berry MJ (2015) High accuracy decoding of dynamical motion from a large retinal population. PLoS Comput Biol 11:e1004304.

McDermott J, Weiss Y, Adelson EH (2001) Beyond junctions: nonlocal form constraints on motion interpretation. Perception 30:905-923.

Morrie RD, Feller MB (2016) Development of synaptic connectivity in the retinal direction selective circuit. Curr Opin Neurobiol 40:45-52.

Nowak P, Dobbins AC, Gawne TJ, Grzywacz NM, Amthor FR (2011) Separability of stimulus parameter encoding by on-off directionally selective rabbit retinal ganglion cells. J Neurophysiol 105:2083-2099.

Olveczky B, Baccus S, Meister M (2003) Segregation of object and background motion in the retina. Nature 423:401-408.

Oyster C (1968) The analysis of image motion by the rabbit retina. J Physiol 199:613-635.

Oyster CW, Barlow HB (1967) Direction-selective units in rabbit retina: distribution of preferred directions. Science 155:841-842.

Panzeri S, Macke JH, Gross J, Kayser C (2015) Neural population coding: combining insights from microscopic and mass signals. Trends Cogn Sci 19:162-172.

Perge J, Borghuis B, Bours R, Lankheet M, van Wezel R (2005) Temporal dynamics of direction tuning in motion-sensitive macaque area MT. J Neurophysiol 93:2104-2116.

Petrusca D, Grivich M, Sher A, Field G, Gauthier J, Shlens J, Chichilnisky E, Litke A (2005) Physiological characterization of a new macaque retinal ganglion cell class. Soc Neurosci Abstr 31.

Pitkow X, Meister M (2012) Decorrelation and efficient coding by retinal ganglion cells. Nat Neurosci 15:628-635.

Pouget A, Dayan P, Zemel R (2000) Information processing with population codes. Nat Rev Neurosci 1:125-132.
Ravi S, Ahn D, Greschner M, Chichilnisky EJ, Field GD (2018) Pathway-specific asymmetries between $\mathrm{ON}$ and OFF visual signals. J Neurosci 38:9728-9740.

Rivlin-Etzion M, Zhou K, Wei W, Elstrott J, Nguyen P, Barres B, Huberman A, Feller M (2011) Transgenic mice reveal unexpected diversity of on-off direction-selective retinal ganglion cell subtypes and brain structures involved in motion processing. J Neurosci 31:8760-8769.

Rust N, Movshon J (2005) In praise of artifice. Nat Neurosci 8:1647-1650.

Sabbah S, Gemmer JA, Bhatia-Lin A, Manoff G, Castro G, Siegel JK, Jeffery N, Berson DM (2017) A retinal code for motion along the gravitational and body axes. Nature 546:492-497.

Salinas E, Abbott LF (1994) Vector reconstruction from firing rates. J Comput Neurosci 1:89-107.

Schneidman E, Bialek W, Berry M (2003) Synergy, redundancy, and independence in population codes. J Neurosci 23:11539-11553.

Schwartz G, Taylor S, Fisher C, Harris R, Berry M (2007) Synchronized firing among retinal ganglion cells signals motion reversal. Neuron 55:958-969.

Shamir M (2014) Emerging principles of population coding: in search for the neural code. Curr Opin Neurobiol 25:140-148.

Shamir M, Sompolinsky H (2004) Nonlinear population codes. Neural Comput 16:1105-1136.

Shlens J, Field G, Gauthier J, Grivich M, Petrusca D, Sher A, Litke A, Chichilnisky E (2006) The structure of multi-neuron firing patterns in primate retina. J Neurosci 26:8254-8266.

Simoncelli E, Olshausen B (2001) Natural image statistics and neural representation. Annu Rev Neurosci 24:1193-1216.

Simpson JI, Leonard CS, Soodak RE (1988) The accessory optic system. Analyzer of self-motion. Ann NY Acad Sci 545:170-179.

Sung K, Wojtach WT, Purves D (2009) An empirical explanation of aperture effects. Proc Natl Acad Sci USA 106:298-303.

Theunissen F, Miller J (1991) Representation of sensory information in the cricket cercal sensory system. II. Information theoretic calculation of system accuracy and optimal tuning-curve widths of four primary interneurons. J Neurophysiol 66:1690-1703.

Trenholm S, Johnson K, Li X, Smith R, Awatramani G (2011) Parallel mechanisms encode direction in the retina. Neuron 71:683-694.

Trenholm S, Schwab D, Balasubramanian V, Awatramani G (2013) Lag normalization in an electrically coupled neural network. Nat Neurosci 16:154-156

Van der Steen J, Collewijn H (1984) Ocular stability in the horizontal, frontal and sagittal planes in the rabbit. Exp Brain Res 56:263-274.

van Hateren J, van der Schaaf A (1998) Independent component filters of natural images compared with simple cells in primary visual cortex. Proc Biol Sci 265:359-366.

Vaney D (1994) Territorial organization of direction-selective ganglion cells in rabbit retina. J Neurosci 14:6301-6316.

Vaney DI, Sivyer B, Taylor WR (2012) Direction selectivity in the retina: symmetry and asymmetry in structure and function. Nat Rev Neurosci 13:194-208.

Vaney DI, He S, Taylor WR, Levick WR (2001) Computation, neural, and ecological constraints. In: Motion vision (Zanker JM, ZeilJ, eds). Berlin: Springer.

Wallace DJ, Greenberg DS, Sawinski J, Rulla S, Notaro G, Kerr JN (2013) Rats maintain an overhead binocular field at the expense of constant fusion. Nature 498:65-69.

Warland D, Reinagel P, Meister M (1997) Decoding visual information from a population of retinal ganglion cells. J Neurophysiol 78:2336-2350.

Yao X, Cafaro J, McLaughlin AJ, Postma FR, Paul DL, Awatramani G, Field GD (2018) Gap junctions contribute to differential light adaptation across direction-selective retinal ganglion cells. Neuron 100:216-228.e6.

Zylberberg J (2018) The role of untuned neurons in sensory information coding. bioRxiv. 134379. doi.org/10.1101.134379.

Zylberberg J, Cafaro J, Turner M, Shea-Brown E, Rieke F (2016) Directionselective circuits shape noise to ensure a precise population code. Neuron 89:369-383. 